\title{
A Theory of Multi-perspective Defocusing
}

\author{
Yuanyuan Ding \\ University of Delaware \\ ding@eecis.udel.edu
}

\author{
Jing Xiao \\ Epson R\&D, Inc. \\ xiaoj@erd.epson.com
}

\author{
Jingyi Yu \\ University of Delaware \\ yu@eecis.udel.edu
}

\begin{abstract}
We present a novel theory for characterizing defocus blurs in multi-perspective cameras such as catadioptric mirrors. Our approach studies how multi-perspective ray geometry transforms under the thin lens. We first use the General Linear Cameras (GLCs) [21] to approximate the incident multi-perspective rays to the lens and then apply a Thin Lens Operator (TLO) to map an incident GLC to the exit GLC. To study defocus blurs caused by the GLC rays, we further introduce a new Ray Spread Function (RSF) model analogous the Point Spread Function (PSF). While PSF models defocus blurs caused by a $3 D$ scene point, RSF models blurs spread by rays. We derive closed form RSFs for incident GLC rays, and we show that for catadioptric cameras with a circular aperture, the RSF can be effectively approximated as a single or mixtures of elliptic-shaped kernels. We apply our method for predicting defocus blurs on commonly used catadioptric cameras and for reducing defocus blurs in catadioptric projections. Experiments on synthetic and real data demonstrate the accuracy and general applicability of our approach.
\end{abstract}

\section{Introduction}

Defocus blurs are useful photographic techniques as well as a potential class of images suitable for analysis by computer vision. The equations governing defocus blurs are well-known in geometric optics. Given a thin lens with focal length $f$ and aperture diameter $D$ (and thus $f$-number $N=f(D)$, if we assume that the sensor/image plane $\Pi_{I}$ lies at a unit distance away from the lens and the camera focuses at scene depth $d_{s}$, we can compute the size of the blur kernel $b_{p}$ for every scene point $\dot{P}$ at depth $d_{p}$ as:

$$
b_{p}=\alpha \frac{\left|d_{p}-d_{s}\right|}{d_{p}\left(d_{s}-\beta\right)}
$$

where $\alpha=f^{2} / N$, and $\beta=f$.

Since all rays originating from $\dot{P}$ converges at $\dot{P}^{\prime}$ after transmitting through the lens, this is analogous to mapping a pinhole camera with Center-of-Project (CoP) $\dot{P}$ to a dif- ferent pinhole camera with $\mathrm{CoP} \dot{P}^{\prime}$. In this paper, we call this defocusing process perspective defocusing. In contrast, if rays from $\dot{P}$ are first reflected by a curved mirror, the incident rays to the lens generally form a multi-perspective camera [23], and so do the exit rays towards the sensor. We call such defocusing processes multi-perspective defocusing, as shown in Figure 3.

Multi-perspective defocusing commonly exists in catadioptric cameras [11], in which a commodity digital camera is placed in front of specially shaped mirrors to capture a much wider field-of-view. Most catadioptric cameras, however, neglect multi-perspective defocusing by using an ultra-small aperture, i.e., its diameter $D \approx 0$. In practice, applications such as low-light imaging and catadioptric projection $[5,20]$ often require using a wide aperture for gathering/emitting more light, and very little work has been focused on modeling and reducing defocus blurs in catadioptric systems.

In this paper, we present a novel theory for characterizing multi-perspective defocusing. Our theory builds upon ray geometry analysis: we study how multi-perspective ray geometry transforms through a thin lens. We parameterize the rays using a two-plane-parametrization (2PP) [10, 6] and use the General Linear Cameras (GLCs) [21] to first approximate the incident multi-perspective rays to the lens. We then derive a Thin Lens Operator (TLO) to map the incident GLC to the exit GLC. Based on the TLO, we derive a slit-direction/slit-slit duality theorem and we show that an incident XSlit GLC [25] always transforms to an exit XSlit or pushbroom GLC [7].

To study defocus blurs caused by the GLC rays, we introduce a new Ray Spread Function (RSF) model analogous the Point Spread Function (PSF). While PSF models defocus blurs caused by a 3D scene point, RSF models blurs spread by rays. We derive closed-form RSFs for incident GLC rays, and we show that for catadioptric cameras with a circular aperture, the RSF can be effectively approximated as a single or mixtures of elliptic-shaped kernels. We apply our method for predicting defocus blurs on commonly used catadioptric cameras and for reducing defocus blurs in catadioptric projectors. Experiments on synthetic and real 
data demonstrate the accuracy and general applicability of our approach.

\section{Related Work}

Our work is motivated by recent advances in defocus analysis, ray geometry analysis, and catadioptric camera/projector designs.

Defocus Blurs. The causes of defocus blurs are well documented in computer vision and photography. Tremendous efforts have been focused on developing robust and effective techniques for reducing blurs [9] and on using blurs for recovering scene depth [8]. Recent work in computational photography suggests that it is beneficial to analyze defocus blurs under specially designed aperture. Coded apertures[19, 4, 24], for example, correlate the frequency characteristics of the blur kernel with scene depth and apply special deconvolution algorithms to simultaneously reduce blurs and recover the scene. These methods are all based on the perspective defocusing model and cannot be easily extended to multi-perspective imaging systems.

Ray Geometry Analysis. Our framework builds upon ray geometry analysis. Rays are directed lines in 3D space. They represent the visual information about a scene by their associated radiance function. Recent studies in camera modeling [14] and distortion analysis [18] have shown that when rays follow specific geometric structures, they and their associated radiance provide a precise definition of a projected image of 3D scene [1]. For example, Yu and McMillan developed a theoretical framework called the General Linear Cameras or GLCs [21] to uniformly model multi-perspective camera models in terms of the planar ray structures. Swamingnathan and Nayar proposed to use the envelop of these rays called the caustic surfaces to characterize distortions [17]. In this paper, we investigate how ray geometry transforms through a thin lens.

Catadioptric Systems. Finally, our framework aims to assist catadioptric camera/projector designs. A catadioptric camera combines a commodity camera with curved mirrors to achieve ultra-wide FoV. Classical examples include single viewpoint catadioptric sensors based on hyperbolic or parabolic mirrors [2] and multiple viewpoint sensors based on spherical, conical, and equiangular mirrors [3]. Nearly all these systems assume that the view camera is pinhole. In reality, it is often desirable to use a wide aperture to gather sufficient light. However, very little work has been focused on analyzing defocus blurs in catadioptric systems. Two exceptions are catadioptric projectors $[5,20]$ which combines a projector with mirrors and the caustic-based catadioptric defocusing analysis [16]. In [5, 20], the authors propose to approximate deblurs using the light transport matrix. We show that our multi-perspective defocusing analysis provides an alternative but more directly method to model defocus blurs and to compensate blurs. In [16], the author uses

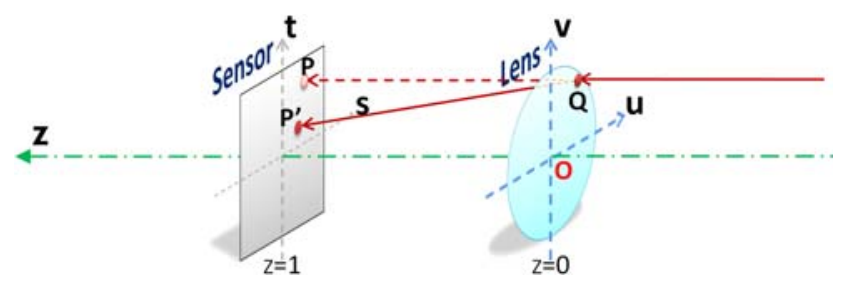

Figure 1. Our analysis uses the in-lens ray parametrization $[u, v, s, t]$.

caustic analysis to study where the lens should focus to capture clear reflection images. Our approach, in contrast, focuses on characterizing the causes of multi-perspective defocusing and on predicting blur kernels.

\section{Ray Geometry Through A Thin Lens}

To analyze multi-perspective defocus blurs, we start with studying how a thin lens transforms ray geometry. To parameterize the rays, we use thin-lens two plane parametrization: we choose the aperture plane as the $u v$-plane at $z=0$ and the image sensor plane as the $s t$-plane at $z=1$. Each ray is parameterized by its intersection point with the two planes as $[u, v, s, t]$, as shown in Figure 1.

\subsection{The Thin Lens Operator (TLO)}

The TLO $L(\circ)$ maps an incident ray $\vec{r}=[u, v, s, t]$ to the lens to the exit ray $\overrightarrow{r^{\prime}}=\left[u^{\prime}, v^{\prime}, s^{\prime}, t^{\prime}\right]$ towards the sensor. Under the thin lens assumption, we have $u^{\prime}=u, v^{\prime}=v$. We can then use the similitude relationship to find $s^{\prime}$ and $t^{\prime}$ as:

$\left[u^{\prime}, v^{\prime}, s^{\prime}, t^{\prime}\right]=L([u, v, s, t])=\left[u, v, s-\frac{1}{f} u, t-\frac{1}{f} v\right]$

The TLO hence is a linear, or more precisely, a shear operator to the $[u, v, s, t]$ ray coordinate. Similar derivations have been shown in $[12,15]$ for analyzing the light field.

\subsection{Duality between Slits and Directions}

Next, we study how the TLO transforms ray geometry. We assume that the lens has focal length $f$ and has two focal planes: $\Pi_{L-}$ at $z=-f$ on the world side and $\Pi_{L+}$ at $z=f$ on the sensor side. We call all rays approaching the lens from the world the incident rays and the ones leaving the lens towards the sensor the exit rays.

Theorem 1 (Slit-Slit Duality). If all incident rays pass through a slit l that does not lie on $\Pi_{L_{-}}$, then all exit rays will pass through a different slit $l^{\prime}$.

Proof. We distinguish the following two cases:

(i) $l$ is parallel to $\Pi_{L-}$ : We can parameterize $l$ using a point $\left[x_{0}, y_{0}, z_{0}\right]$ on $l$ and the direction $\left[d^{x}, d^{y}, 0\right]$ of $l$. All rays $[u, v, s, t]$ passing through $l$ satisfy:

$[u, v, 0]+\lambda_{1}[s-u, t-v, 1]=\left[x_{0}, y_{0}, z_{0}\right]+\lambda_{2}\left[d^{x}, d^{y}, 0\right]$ 
It is easy to verify that $\lambda_{1}=z_{0}$ and $z_{0} \neq-f$. We can then rewrite Eqn. (3) in $\left[u^{\prime}, v^{\prime}, s^{\prime}, t^{\prime}\right]$ using the TLO as:

$\left[u^{\prime}, v^{\prime}, 0\right]+\gamma z_{0}\left[s^{\prime}-u^{\prime}, t^{\prime}-v^{\prime}, 1\right]=\gamma\left[x_{0}, y_{0}, z_{0}\right]+\gamma \lambda_{2}\left[d^{x}, d^{y}, 0\right]$

where $\gamma=\frac{f}{f+z_{0}}$. This indicates that all exit rays will pass through a slit $l^{\prime}$ parameterized by a point $\frac{f}{f+z_{0}}\left[x_{0}, y_{0}, z_{0}\right]$ and its direction $\left[d^{x}, d^{y}, 0\right]$.

(ii) $l$ is not parallel to $\Pi_{L-}$ : Therefore $l$ will intersect the $u v$-plane at $\left[u_{0}, v_{0}, 0\right]$ and the $s t$-plane at $\left[s_{0}, t_{0}, 1\right]$. All rays passing through $l$ satisfy the bilinear constraint [21]:

$$
\left(u-u_{0}\right)\left(t-t_{0}\right)-\left(v-v_{0}\right)\left(s-s_{0}\right)=0
$$

Rewriting Eqn.(3) in $\left[u^{\prime}, v^{\prime}, s^{\prime}, t^{\prime}\right]$ using the TLO, we have:

$$
\left(u^{\prime}-u_{0}\right)\left(t^{\prime}-t_{0}+\frac{v_{0}}{f}\right)-\left(v^{\prime}-v_{0}\right)\left(s^{\prime}-s_{0}+\frac{u_{0}}{f}\right)=0
$$

Eqn.(5) indicates that all exit rays will pass through a line $l^{\prime}$ that intersects the $u v$-plane at $\left[u_{0}, v_{0}, 0\right]$ and the $s t$-plane at $\left[s_{0}-\frac{u_{0}}{f}, t_{0}-\frac{v_{0}}{f}, 1\right]$.

Theorem 1 reveals that the TLO preserves the slit-type ray geometry if the slit does not lie on the lens' focal plane $\Pi_{L-\text {. }}$

Theorem 2 (Slit-Direction Duality). If all incident rays pass through a line $l$ that lies on $\Pi_{L-}$, then all exit rays will be parallel to the plane formed by $l$ and the lens optical center $\dot{O}$.

Proof. Notice that we can parameterize $l$ with a point $\dot{P}=$ $\left[x_{0}, y_{0},-f\right]$ on $l$ and the direction $\left[d^{x}, d^{y}, 0\right]$ of $l$. Therefore, all rays passing through $l$ satisfy:

$[u, v, 0]-f[s-u, t-v, 1]=\left[x_{0}, y_{0},-f\right]+\lambda_{2}\left[d^{x}, d^{y}, 0\right]$

Eliminating $\lambda_{2}$, we have:

$$
\frac{u-f(s-u)-x_{0}}{v-f(t-v)-y_{0}}=\frac{d^{x}}{d^{y}}
$$

We can then use the thin lens operator to substitute $[s, t, u, v]$ with $\left[s^{\prime}, t^{\prime}, u^{\prime}, v^{\prime}\right]$ and we have:

$\left(s^{\prime}-u^{\prime}, t^{\prime}-v^{\prime}, 1\right)^{T} \cdot\left(-f d^{y}, f d^{x}, y_{0} d^{x}-x_{0} d^{y}\right)^{T}=0$

Eqn.(7) reveals that all exit rays are orthogonal to vector $\vec{n}$ :

$\vec{n}=\left(-f d^{y}, f d^{x}, y_{0} d^{x}-x_{0} d^{y}\right)^{T}=\left(d^{x}, d^{y}, 0\right)^{T} \otimes\left(x_{0}, y_{0},-f\right)^{T}$

where $\otimes$ is the cross product. We can further verify that $\vec{n}$ is the normal direction of the plane formed by $l$ and the lens optical center $\dot{O}$.

Theorem 2 proves the slit-direction duality through the thin lens, i.e., the lens maps a slit to a direction if the slit lies on the focal plane. It also provides an efficient way to find the direction.

Theorem 3 (Direction-Slit Duality). If all incident rays are parallel to some plane $\Pi$ through the optical center $O$, then

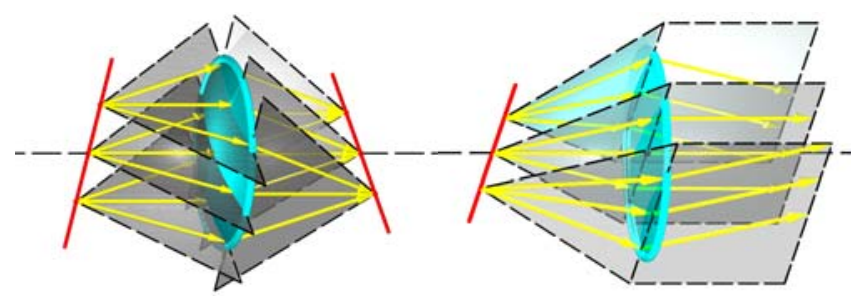

Figure 2. Slit-Direction Duality. When the slit of a GLC lies on the lens' focal plane, a pushbroom transforms to a different pushbroom (left) and a pencil transforms to a twisted orthographic (right).

all exit rays will pass through a slit $l$ that is parallel to the $2 P P$ and lies on $\Pi_{L+}$. Specifically, we can find l intersecting $\Pi$ with plane $\Pi_{L+}$.

The proof follows reciprocity of rays and Theorem 2 . Fig.2 illustrates the slit-slit and slit-direction duality.

\subsection{GLC Through A Thin Lens}

Next, we study how general multi-perspective ray geometry transforms through the thin lens. We use the recently proposed General Linear Camera (GLC) model to approximate the ray geometry. A GLC collects affine combinations of three generator rays parameterized under $2 \mathrm{PP}$ :

$$
\mathrm{GLC}:=\left\{\vec{r} \mid \vec{r}=\alpha \cdot \vec{r}_{1}+\beta \cdot \vec{r}_{2}+(1-\alpha-\beta) \cdot \vec{r}_{3}\right\}
$$

Theorem 4. A incident GLC transforms to an exit GLC through the thin lens.

Since $L(\circ)$ is a linear operator, for every incident ray $\vec{r}$, we can compute its exit ray as:

$$
\begin{aligned}
L(\vec{r}) & =L\left(\alpha \vec{r}_{1}+\beta \vec{r}_{2}+(1-\alpha-\beta) \vec{r}_{3}\right) \\
& =\alpha L\left(\vec{r}_{1}\right)+\beta L\left(\vec{r}_{2}\right)+(1-\alpha-\beta) L\left(\vec{r}_{3}\right)
\end{aligned}
$$

Eqn.(9) reveals that the exit rays also form a GLC where the three new generator rays are $L\left(r_{1}\right), L\left(r_{2}\right)$, and $L\left(r_{3}\right)$.

Next, we consider how GLC ray geometry transforms through the thin lens. There are precisely eight GLCs: in a pinhole camera, all rays pass through a single point; in an orthographic camera, all rays are parallel; In a pushbroom camera [7], all rays lie on a set of parallel planes and pass through a line; in a XSlit camera [25], all rays pass through two non-coplanar lines; in a pencil camera, all coplanar rays originate from a point on a line and lie on a specific plane through the line; in a twisted orthographic camera, all rays lie on parallel twisted planes and no rays intersect; in an bilinear camera [13], no two rays are coplanar and no two rays intersect; and in an EPI camera, all rays lie on a 2D plane. Except for the degenerate case of the EPI, we enumerate how TLO transforms each type of GLCs:

I. XSlit: For XSlit cameras, we discuss two cases: (a) one of the two slits lies on $\Pi_{L-}$, or (b) neither slits lies on $\Pi_{L-}$. 


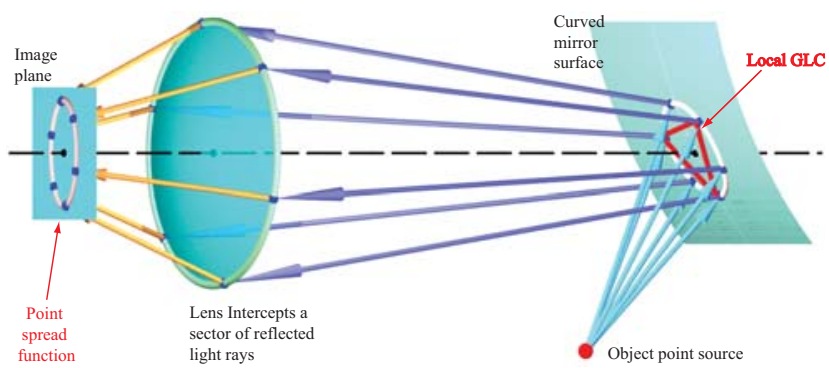

Figure 3. Defocus Analysis on a Catadioptric Mirror. We approximate the reflection rays from a scene point as an incident GLC and then compute its Ray Spread Function on the image.

Denote $\lambda_{1}$ and $\lambda_{2}$ the depths of the two slits $l_{1}$ and $l_{2}$ respectively.

(a) Assume $l_{1}$ lies on $\Pi_{L-}$ and $l_{2}$ does not. We have, $\lambda_{1}=-f$ and $\lambda_{2} \neq-f$, by Theorem 1 and 2 , we have that all exit rays will pass through some line $l_{2}^{\prime}$ and will be parallel to the plane determined by $l_{1}$ and lens optical center $\dot{O}$. Therefore, the exit GLC is a pushbroom.

(b) Since neither slits lie on $\Pi_{L-}$, we have $\lambda_{1} \neq-f$ and $\lambda_{2} \neq-f$. By Theorem 1, all exit rays will pass through two distinct slits $l_{1}^{\prime}$ and $l_{2}^{\prime}$. Therefore, the exit GLC is a XSlit.

II. Pushbroom: A pushbroom camera collects rays that pass through a slit $l$ and are parallel to some plane $\Pi$ passing through the optical center $O$.

(a) If $l$ lies on $\Pi_{L_{-}}$, by Theorem 2 , all exit rays are parallel to the plane determined by $l$ and lens optical center $\dot{O}$. By Theorem 3, all exit rays will pass through the slit $l^{\prime}$. Therefore, the exit GLC is still a pushbroom.

(b) If $l$ does not lie on $\Pi_{L-}$, by Theorem 1 , all exit rays will pass through a new slit $l_{1}^{\prime}$. By Theorem 3, we also have that the direction of the rays will map to a second slit $l_{2}^{\prime}$. Therefore, the exit GLC is a XSlit.

\section{Pinhole:}

(a) If the CoP $\dot{C}$ of the camera does not lie on $\Pi_{L-}$, then all exit rays will pass through some point $\dot{C}^{\prime}$. Therefore, the exit GLC is a pinhole.

(b) If $\dot{C}$ lies on $\Pi_{L-}$, then the exit GLC is an orthographic.

IV. Pencil: A pencil camera collects rays on a set of nonparallel planes that share a line $l$.

(a) If $l$ does not lie on $\Pi_{L-}$, we have $\lambda \neq-f$, by Theorem 1 , all exit rays will pass through a line $l^{\prime}$. And rays that intersect at the same point $\dot{Q}$ on $l$ will still intersect at some point $\dot{Q}^{\prime}$ on $l^{\prime}$. Therefore, the exit GLC is still a pencil.

(b) When $l$ lies on $\Pi_{L-}$, by Theorem 2 all exit rays will be parallel to the plane determined by $l$ and lens optical center $\dot{O}$. Therefore, the exit GLC is a twisted orthographic.

V. Bilinear: In a bilinear camera, every pair of rays are oblique. By Theorem 1, it is easy to verify that the exit rays will satisfy the same constraint. Therefore, the exit GLC is

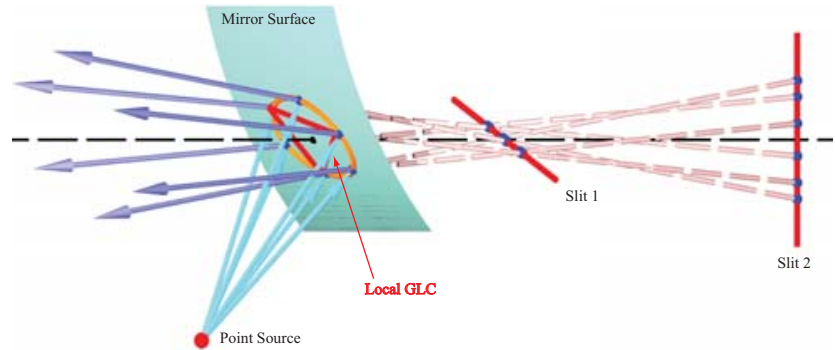

Figure 4. A patch of reflection rays form a XSlit GLC.

also a bilinear.

VI. Orthographic: An orthographic camera always maps to a pinhole camera.

VII. Twisted Orthographic: A twisted orthographic camera collect rays that are parallel to a set of parallel planes. By duality with respect to the pencil camera, we have that the exit GLC is a pencil.

\section{Multi-perspective Defocus Analysis}

To more precisely define multi-perspective defocusing, we first review perspective defocusing: all rays emitting from a 3D scene point will first converge at a different 3D point through the thin lens; the cone of rays will spread onto a disk of pixels on the sensor. In classical photography, this process is commonly described using the Point Spread Function (PSF), i.e., the mapping from a 3D point to pixels. Notice that PSF can be alternatively viewed as mapping an incident pinhole GLC to pixels. Therefore, we introduce a new Ray Spread Function or RSF model to describe how a general set of incident rays spread to pixels on the sensor. The classical PSF is a special case of the RSF when the incident rays form a pinhole GLC. Our goal is to study the RSFs of incident GLCs.

For general multi-perspective incident rays, we can first decompose the rays into piecewise GLCs and compute the RSF for each individual GLC. For example, on a catadioptric mirror, we can parameterize the mirror surface as $z(x, y)$ with respect to the $u v$ plane. We can then approximate the mirror surface as a triangle mesh. At each vertex $(x, y)$, we compute the reflection ray from the scene point $\dot{P}$ as:

$$
[u(x, y), v(x, y), s(x, y), t(x, y)]=R(z(x, y), \dot{P})
$$

where $R$ is the reflection operator. The reflection ray triplet on each triangle then maps to an incident GLC, as shown in Fig. 3.

\subsection{The RSFs of GLCs}

The Aperture Constraint. To derive the RSF of an incident GLC, we begin with studying the role of the aperture. Recall that the aperture blocks part of the incident GLC rays. Therefore, we define the aperture using a constraint 


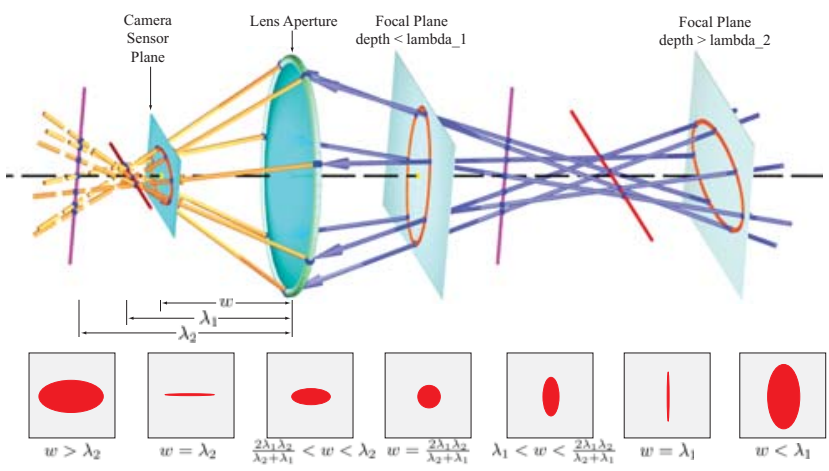

Figure 5. The RSF of a XSlit GLC. The shape of the RSF is generally elliptic whose major and minor radii are functions of the depths of the two slits.

function $G$ on the $u v$ aperture plane: a ray $r(u, v, s, t)$ can pass through the aperture if $G(u, v) \leq 0$. For example, the constraint function of a circular aperture of diameter $D$ is:

$$
G(u, v)=u^{2}+v^{2}-D / 2^{2}
$$

Next, we consider the RSF of an incident GLC. We first use the thin lens operator (TLO) to map the incident GLC to the exit GLC, as shown in Section 3.3. For clarity, we use $\left(u, v, s^{\prime}, t^{\prime}\right)$ to represent rays in the exit GLC so that $\left(s^{\prime}, t^{\prime}\right)$ directly represents the pixel coordinate on the sensor. Our goal is to transform the aperture constraint $G(u, v)$ to pixel constraint $G\left(s^{\prime}, t^{\prime}\right)$. This requires computing $u$ and $v$ in terms of $s^{\prime}$ and $t^{\prime}$ using the GLC constraints.

Recall that a GLC collects rays that lie on the 2D affine subspace in the $4 \mathrm{D}$ ray space. Therefore, we can rewrite GLC in terms of two linear constraints:

$$
u=\phi_{1} s^{\prime}+\phi_{2} t^{\prime}+\phi_{3}, \quad v=\phi_{4} s^{\prime}+\phi_{5} t^{\prime}+\phi_{6}
$$

We can substitute Eqn.(12) into the aperture constraint as:

$$
G(u, v)=G\left(\phi_{1} s^{\prime}+\phi_{2} t^{\prime}+\phi_{3}, \phi_{4} s^{\prime}+\phi_{5} t^{\prime}+\phi_{6}\right)
$$

Eqn.(13) imposes a new constraint to the pixels $\left(s^{\prime}, t^{\prime}\right)$ on the sensor and hence defines the size and shape (i.e., the spread) of the blur kernel.

If we further use a circular shaped aperture, we can substitute Eqn.(12) into Eqn.(11) and we have:

$$
\left(\phi_{1} s^{\prime}+\phi_{2} t^{\prime}+\phi_{3}\right)^{2}+\left(\phi_{4} s^{\prime}+\phi_{5} t^{\prime}+\phi_{6}\right)^{2} \leq\left(\frac{D}{2}\right)^{2}
$$

Equation (14) reveals that the RSF of a GLC is ellipticshaped. For general multi-perspective incident rays, since we can approximate them incident rays into piecewise GLCs, their RSF should have the shape of mixtures of ellipses.

\subsection{A Case Study: The RSF of A XSlit GLC}

Next, we focus on studying a special XSlit GLC. We assume that neither slits of the GLC lies on the focal plane of the lens. By Theorem 4, the exit GLC is a also XSlit with

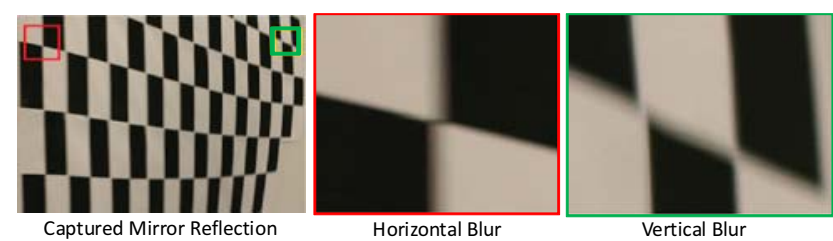

Figure 6. Defocus Blurs on a Cylindrical Mirror. We capture a reflection image (left) of a checkerboard on a cylindrical mirror. Notice how the blur directions transition from mostly horizontal (middle) to mostly vertical (right).

two slits $l_{i}, i=1,2$ that lie at depth $z=\lambda_{1}$ and $z=\lambda_{2}$ respectively, as shown in Figure 5.

To simplify our analysis, we consider the special case when the slits have orthogonal directions. We rotate the coordinate system so that the slit directions align with the $u$ and $v$ axis. The resulting exit GLC satisfies:

$$
\left\{\begin{array} { l } 
{ ( 1 - \lambda _ { 1 } ) u + \lambda _ { 1 } s ^ { \prime } = 0 } \\
{ ( 1 - \lambda _ { 2 } ) v + \lambda _ { 2 } t ^ { \prime } = 0 }
\end{array} \Longrightarrow \left\{\begin{array}{l}
u=\frac{s^{\prime}}{1-\frac{1}{\lambda_{1}}} \\
v=\frac{t^{\prime}}{1-\frac{1}{\lambda_{2}}}
\end{array}\right.\right.
$$

Substitute Eqn. (15) into the circular aperture function Eqn. (11), we have:

$$
\left(\frac{s^{\prime}}{\frac{1}{\lambda_{1}}-1}\right)^{2}+\left(\frac{t^{\prime}}{\frac{1}{\lambda_{2}}-1}\right)^{2} \leq\left(\frac{D}{2}\right)^{2}
$$

Eqn. (16) reveals that the major and minor radii of the elliptic defocus kernel are $\left|\frac{1}{\lambda_{1}}-1\right|$ and $\left|\frac{1}{\lambda_{2}}-1\right|$. We can further elaborate on various cases for different $\lambda_{1}$ and $\lambda_{2}$, as shown in Fig. 5.

(i) When $\frac{2 \lambda_{1} \lambda_{2}}{\lambda_{2}+\lambda_{1}}>1$, the major radius is $\left|\frac{1}{\lambda_{2}}-1\right| \cdot \frac{D}{2}$ and has the same direction of $l_{1}$.

(ii) When $\lambda_{1}=1$, the RSF degenerates to a line segment (a 1D RSF) whose length is $\left|\frac{1}{\lambda_{2}}-1\right| \cdot \frac{D}{2}$. This should not be surprising because $l_{1}$ lies on the sensor plane.

(iii) When $\frac{2 \lambda_{1} \lambda_{2}}{\lambda_{2}+\lambda_{1}}=1$, the shape of the RSF becomes a circular disk where the radius of the disk is $\left|\frac{\lambda_{1}-\lambda_{2}}{\lambda_{1}+\lambda_{2}}\right| \cdot \frac{D}{2}$.

(iv) When $\frac{2 \lambda_{1} \lambda_{2}}{\lambda_{2}+\lambda_{1}}<1$, the major radius is $\left|\frac{1}{\lambda_{1}}-1\right| \cdot \frac{D}{2}$ and has the same direction of the second slit $l_{2}$.

(iv) When $\lambda_{2}=1$, the second slit lies on the sensor plane and RSF degenerates to a line segment (a 1D RSF) whose length is $\left|\frac{1}{\lambda_{2}}-1\right| \cdot \frac{D}{2}=0$.

This analysis is particularly useful as it has been shown in [22] that local reflection rays from a $3 \mathrm{D}$ point can be effectively approximated as a XSlit camera. Therefore, the RSF caused by a 3D scene point in a catadioptric mirror can only be an ellipse, a circle, or a line segment. Furthermore, the shape of the RSF depends on the location of the scene point. To verify our analysis, we capture an reflection image on a curved cylindrical mirror. We put a checkerboard in 3D space and capture the image with a Canon DSLR camera with an EF 50mm lens of f-number 1.8, as shown in Fig.6. 
Notice the defocus blurs are anisotropic in the captured image. For example, the blur direction is mostly horizontal on the left part of the image and transitions to vertical at the right part of the image.

\section{Applications}

Finally, we apply our multi-perspective defocusing analysis to two applications: RSF prediction on commonly used catadioptric mirrors and defocus compensation on catadioptric projectors

\subsection{RSF Prediction}

Given the mirror surface, the view camera, and the 3D scene, we aim to predict the shape and the size of the defocus blur kernel at every pixel on the image. A brute-force approach is to apply ray-tracing and then analyze the rendered image. We, in contrast, directly predict the blur kernel. For simplicity, our algorithm assumes using a circular shaped aperture on the view camera although it can easily be extended to handle more general cases.

For every pixel $q\left(q^{x}, q^{y}\right)$ in the view camera, we first trace out a ray from $q$ to the lens optical center. We intersect the ray with the mirror, compute its reflected ray, and find its intersection point with the scene as $\dot{Q}$. This process emulates forward ray-tracing on a pinhole camera. Next, we find all reflection rays that originate from $\dot{Q}$ and pass through the lens aperture and approximate them as a GLC. To do so, we trace out three additional rays from $q$ to the rim of the lens. We forward trace these three rays using the TLO and intersect them with the mirror surface at points $\dot{P}_{1}, \dot{P}_{2}$, and $\dot{P}_{3}$. We then compute the three reflected rays with respect to $\dot{Q}$ and construct an incident GLC. Finally, we use the TLO to map the incident GLC to the exit GLC and apply our blur kernel estimation algorithm (Section 4) to compute the RSF.

To validate our algorithm, we compare our predicted RSFs with the ray tracing results. In Figure 7, we illustrate our estimations on both cylindrical and spherical mirrors. We purposely tilt the view camera to show spatially-variant defocus blurs. The ray tracing results are obtained by using the Pov-Ray with a wide aperture. Specifically, we put a plane with dot patterns in the scene and trace out 256 rays per pixel. The ray tracing results are shown in the second and the fourth columns in Fig.7. Next, we apply our RSF estimation and its results are shown in the first and the third columns. Notice that the ray tracing scheme and our estimation methods sample the image plane differently: the sampling grid in ray tracing is in 3D space and therefore it produces non-uniform sampling in the image; our scheme samples a regular grid on the image. Nevertheless, our predictions are highly consistent with the ray-traced results. Notice how defocus kernels change in both shape and size across the image. For example, in the spherical mirror re-

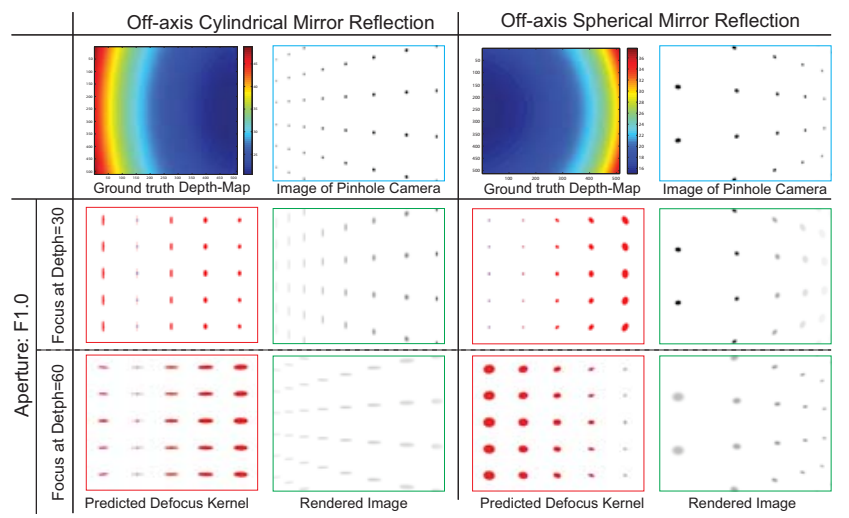

Figure 7. RSF Estimation on Cylindrical Spherical Mirrors. Column 1 and 3 show our estimated RSFs. Column 2 and 4 show the ray tracing results. This figure is best viewed in the electronic version.

sults at focal depth $=30$. The black dots on the left have the same size in both pinhole and wide aperture viewing cameras. This implies that the kernel is rather smaller at those points and our method faithfully predicts the results. In contrast, the dots to the right grow larger in the wide aperture image and our method correctly predicts large blur kernels. Our technique is also much faster than ray tracing: it takes Pov-Ray 40 minutes to render a single wide aperture image (at $256 \times 256$ resolution) whereas our technique predicts the blur kernels in less than a seconds.

We further apply our RSF prediction scheme on three common used catadioptric mirrors: spherical, parabolic, and hyperbolic. Figure 8 shows our RSF prediction results. Recall that the first two mirrors are non-central and the third one is central. We use a plane with dot patterns as scene geometry and set the plane parallel to the camera sensor plane. We gradually change the focus of the view camera in three rows. For a spherical mirror, both the shape and the size of the blur kernels vary across the mirror. As we change the focus of the camera, the defocus kernels change dramatically. Similar phenomenon have been observed in [16]. For a parabolic mirror, the shape and the size of the kernels are more coherent across images. When we change the camera focus closer to the mirror focus, the blur kernel uniformly shrink. In the hyperbolic mirror, if the view camera's CoP is at the mirror focus, the imaging system would resemble a pinhole (central) camera even if we use a wide aperture. This suggest that catadioptric systems based on hyperbolic mirrors are more suitable for imaging applications that require using wide apertures.

\subsection{Catadioptric Projectors}

Finally, we apply our framework for reducing defocus blurs in catadioptric projectors. The recently proposed catadioptric projector [5] combines a commodity projector with curved mirrors to produce ultra-wide FoV projections. 


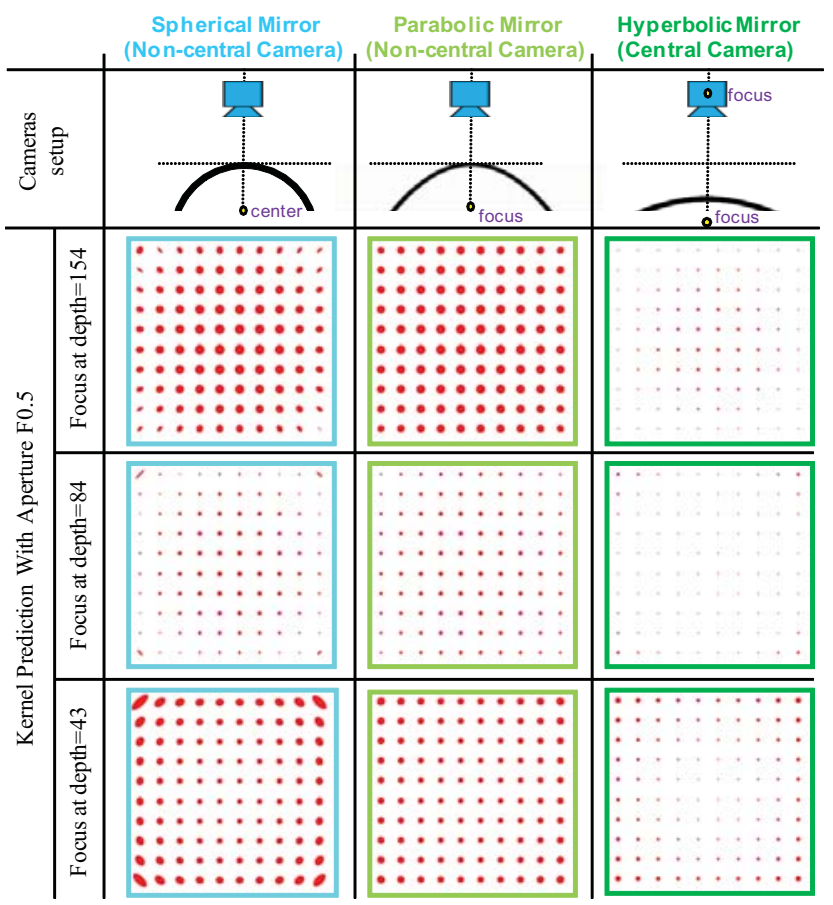

Figure 8. Our Predicted Defocus Blurs on Commonly Used Catadioptric Mirrors. See Section 5.1 for a detailed analysis. This figure is best viewed in the electronic version.

Since a projector relies on wide apertures to produce bright projections, defocus blurs are often more severe, as shown in Figure 9. To model defocus blurs in catadioptric projectors, we treat the project as a dual camera and repeat our analysis our catadioptric cameras.

For experiments, we construct a catadioptric projector by facing a commodity projector (Epson PowerLite 78 projector with resolution $1024 \times 768$ ) towards a cylindrical mirror. Instead of using custom-built mirrors, we use an inexpensive plastic mirror. We bend the mirror to near cylindrical shape to achieve an aspect ratio of 3:1. We assume that the display screen is planar and first validate the RSF estimation scheme.

We first project a grid of dot patterns onto the display screen, where each dot resembles a 3D scene point and its projected image resembles its RSF. Fig.9 (left) illustrates our captured result: the projection image of dots are ellipticshaped and have different sizes and ratios; when we change the focus of the projector, the shape of the blur kernels also change accordingly. This is consistent with our blur kernel analysis in Section 5.1. Furthermore, previous GLC reflection analysis [22] has shown that reflections rays off the cylindrical mirror can be approximated as XSlit GLCs with nearly perpendicular slits. From our derivation in Section 4, we can approximate the kernels as axis-aligned ellipses.

To compensate for defocus blurs, we adopt a hardware solution: we change the shape of the aperture to reduce the average size of the defocus blur kernel. Conceptually, one can use a very small aperture to emulate pinhole-type projection. However, small apertures block a large amount of light and produce dark projections. Our solution is to find the appropriate aperture shape that can effectively reduce the blurs without sacrificing the brightness in projection. Specifically, we search through a class of elliptic-shaped apertures, each of which has the same area as the circle aperture of radius $\frac{D}{2}$.

To find the optimal aperture shape, we reuse the captured projection image of the dot patterns. For each dot, we fit an ellipse to its blur image and save its major and minor radii. We then compute the average major and minor radii across all dots and save them as $a^{\prime}$ and $b^{\prime}$. By reusing our analysis in Section 4, we can verify that the optimal major and minor radii $a$ and $b$ of the aperture correspond to a circular shaped defocus kernel, thus should satisfy $a / b=b^{\prime} / a^{\prime}$. Therefore, we choose $a=\frac{D}{2} \sqrt{a^{\prime} / b^{\prime}}$ and $b=\frac{D}{2} \sqrt{b^{\prime} / a^{\prime}}$. Our analysis is consistent with the observation that if defocus blurs are stronger along one axis, we should reduce the aperture size (length) along that direction. The optimal aperture hence should produce circular-shaped RSFs.

In Fig.9, we compare the panoramic projection results using different aperture shapes. Since the horizontal resolution of our projection is much lower the vertical one, we adjust the focus of the projector (under the circular aperture) to first reduce horizontal blurs. As a result, vertical blurs are much more severe. Next, we project the dot pattern onto the screen and measure the elliptic-shaped blur kernels. Finally, we estimate the optimal aperture and use it in place of the original circular aperture. Fig.9 shows that the use of the new aperture shape significantly reduces defocus blurs. A side effect, however, is that it incurs stronger vignetting artifacts.

\section{Conclusions and Future Work}

We have presented a novel theory for characterizing defocus blurs in multi-perspective cameras. The core of our technique is to study how ray geometry transforms through the thin lens. A major limitation of our framework is that we use the two-plane-parametrization (2PP), which makes our analysis parametrization dependent. One possible solution is to represent the rays and the GLCs using projective geometry [14]. We can then re-formulate the thin lens operator and the RSF without imposing parametrization.

There are a number of future directions that we plan to explore. First, our analysis reveals that the shape of defocus blurs is a function of scene depth. Previous Depth-fromDefocusing (DfD) algorithms have only used the size of the kernel to infer scene geometry. Our theory indicates that additional information such as the kernel shape can be incorporated into the solution. Second, opposite to DfD, if we assume scene geometry is known, our theory may lead a new 


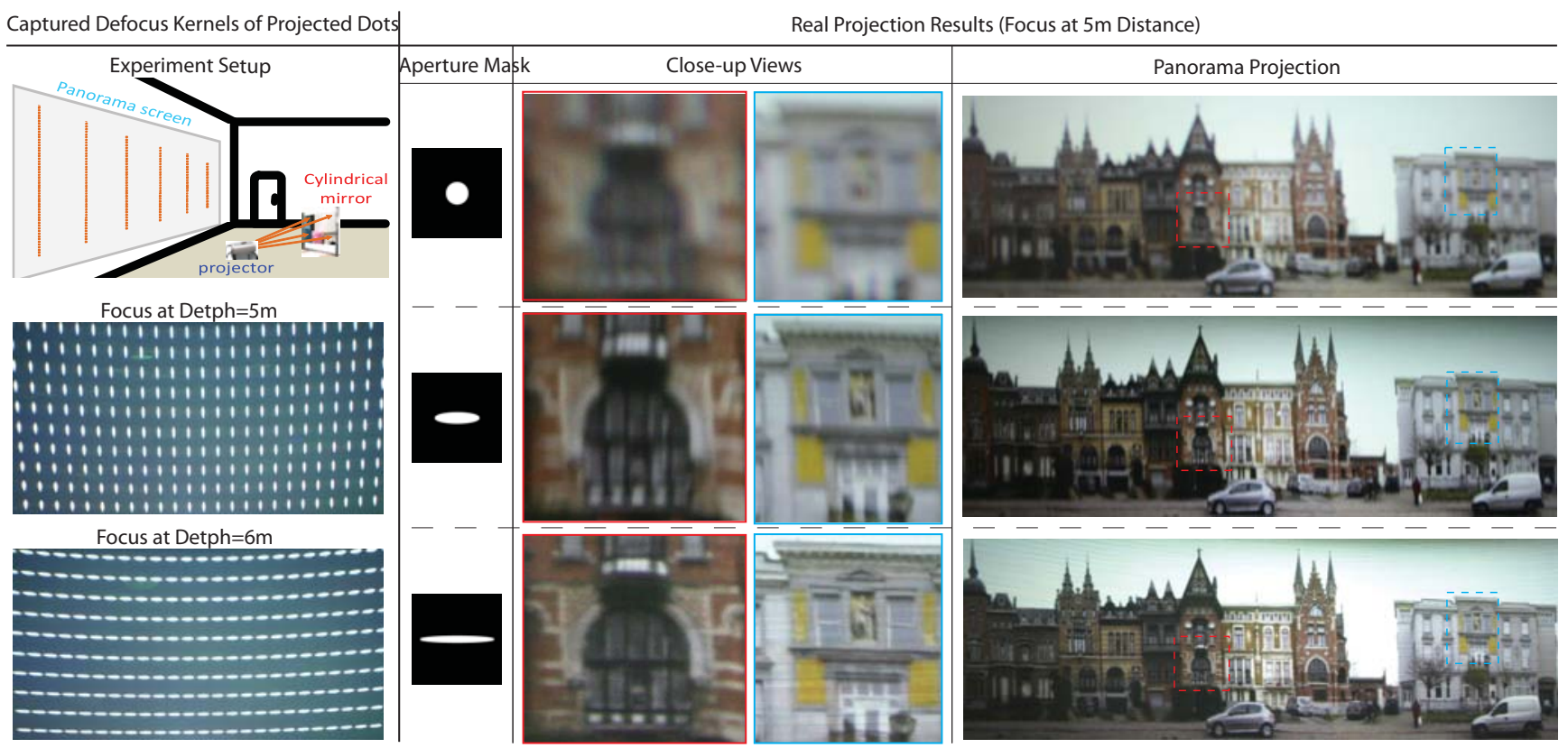

Figure 9. Defocus Compensation in Panoramic Projections. We construct a catadioptric projector using a cylindrical mirror and explore different aperture shapes for reducing blurs.

class of specular surface reconstruction algorithms. For example, we plan to explore new shape-from-blurs techniques by analyzing defocus blurs on mirror or fluid surfaces. Finally, for the problem of blur compensation in catadioptric projectors, we plan to investigate combining the coded aperture technique with our multi-perspective defocusing theory, e.g., to find the optimal coded aperture pattern under catadioptric defocus blurs.

\section{Acknowledgement}

This project was partially supported by the National Science Foundation under grants IIS-CAREER-0845268 and IIS-RI-1016395, and by the Air Force Office of Scientific Research under the YIP Award.

\section{References}

[1] E. H. Adelson and J. R. Bergen. The plenoptic function and the elements of early vision. In Computational Models of Visual Processing, pages 3-20. MIT Press, 1991. 218

[2] S. Baker and S. K. Nayar. A theory of catadioptric image formation. In $I C C V$, pages $35-42,1998.218$

[3] J. Chahl and M. Srinivasan. Reflective surfaces for panoramic imaging. In Applied Optics, 1997. 218

[4] O. Cossairt, C. Zhou, and S. Nayar. Diffusion coded photography for extended depth of field. TOG, 2010. 218

[5] Y. Ding, J. Xiao, K.-H. Tan, and J. Yu. Catadioptric projectors. In CVPR, pages 2528-2535. IEEE, 2009. 217, 218, 222

[6] S. J. Gortler, R. Grzeszczuk, R. Szeliski, and M. F. Cohen. The lumigraph. In SIGGRAPH, 1996. 217

[7] R. Gupta and R. I. Hartley. Linear pushbroom cameras. IEEE TPAMI, 19:963-975, 1997. 217, 219

[8] S. W. Hasinoff and K. N. Kutulakos. Confocal stereo. Int. J. Comput. Vision, 2009. 218
[9] A. Levin, Y. Weiss, F. Durand, and W. Freeman. Understanding and evaluating blind deconvolution algorithms. CVPR, 0:1964-1971, 2009. 218

[10] M. Levoy and P. Hanrahan. Light field rendering. In SIGGRAPH, pages 31-42, 1996. 217

[11] S. K. Nayar. Catadioptric omnidirectional camera. In CVPR, page 482, 1997. 217

[12] R. Ng. Fourier slice photography. In SIGGRAPH, pages 735-744, 2005. 218

[13] T. Pajdla. Stereo with oblique cameras. Int. J. Comput. Vision, 47(13):161-170, 2002. 219

[14] J. Ponce. What is a camera. In CVPR, 2009. 218, 223

[15] C. Soler, K. Subr, F. Durand, N. Holzschuch, and F. Sillion. Fourier depth of field. TOG, 2009. 218

[16] R. Swaminathan. Focus in catadioptric imaging systems. ICCV, 2007. 218,222

[17] R. Swaminathan, M. Grossberg, and S. K. Nayar. Caustics of catadioptric cameras. In ICCV, pages 2-9, 2001. 218

[18] R. Swaminathan, M. Grossberg, and S. K. Nayar. A perspective on distortions. In CVPR, pages 594-601, 2003. 218

[19] A. Veeraraghavan, R. Raskar, A. Agrawal, A. Mohan, and J. Tumblin. Dappled photography: mask enhanced cameras for heterodyned light fields and coded aperture refocusing. In SIGGRAPH, 2007. 218

[20] G. Wetzstein and O. Bimber. Radiometric compensation through inverse light transport. In $P G, 2007.217,218$

[21] J. Yu and L. McMillan. General linear cameras. In ECCV, pages 14-27, 2004. 217, 218, 219

[22] J. Yu and L. McMillan. Modelling reflections via multiperspective imaging. In $C V P R$, pages 117-124, 2005. 221, 223

[23] J. Yu, L. McMillan, and P. Sturm. Multiperspective modeling, rendering, and imaging. In Proceedings of Eurographics, Crete, Greece, April 2008. STAR - State of the Art Report. 217

[24] C. Zhou, S. Lin, and S. Nayar. Coded Aperture Pairs for Depth from Defocus. 2009. 218

[25] A. Zomet, D. Feldman, S. Peleg, , and D. Weinshall. Mosaicing new views: The crossed-slits projection. In IEEE Trans. on PAMI, pages 741-754, 2003. 217, 219 